\title{
Spectral Rendering with Daylight: A Comparison of Two Spectral Daylight Simulation Platforms
}

\author{
Priji Balakrishnan $^{1}$, J. Alstan Jakubiec ${ }^{2}$ \\ ${ }^{1}$ Singapore University of Technology and Design, Singapore \\ ${ }^{2}$ University of Toronto, Toronto, Canada
}

\begin{abstract}
Colours that surround us are not just the result of surface properties, rather the interplay between the spectral distribution of illuminating light and spectrally specific surface reflectance. Despite the temporal and spatial variation of daylight spectral distribution, daylight simulation platforms most commonly use luminance based sky models (CIE or all-weather Perez skies) that lack spectral and colorimetric information. LARK and ALFA are the two currently available spectral daylight simulation platforms that use spectral data of skies and materials to produce daylight renderings. The authors measure and perform visual, spectral and colour difference comparisons of complex urban scenes with different materiality - plaster facades, vegetation, reflective facades - in LARK, ALFA and standard non-spectral daylight simulations. The comparisons present the challenges, applications and limitations of using the currently evolving multi-spectral daylight simulations.
\end{abstract}

\section{Background}

Current daylight simulation platforms are based on three-dimensional colour spaces, such as RGB (Red, Green, Blue), for predictive renderings. Computations in RGB colour space does not suffice the needs of designers when they have to predict colour shifts, colour-dependent lighting metrics (like circadian light), and changing visual perceptions occurring under different sky conditions (Devlin et al., 2002).

Multi-spectral lighting simulation that can also achieve the physical accuracy of light is currently based on the Radiance lighting and visualisation platform (Ward, 1994). Ruppertsberg and Bloj (2006) validated Radiance for colour and luminance accuracy using an N-step method to perform multispectral simulations. The N-step algorithm divides the spectrum into $\mathrm{N}$ consecutive wavebands, and a simulation is performed in each of these $\mathrm{N}$ channels rather than the standard single RGB simulation.

Applications of multi-spectral simulations or spectral rendering platforms are evident in the field of psychophysical analysis, where visual psychophysi- cists need to produce complex simulated stimuli for their experiments. However, these experiments use simulated scenes created using objects and mostly illuminated by artificial sources of light. To accurately determine the colour rendering indices of light sources Geisler-Moroder and Dür (2009) recommends the use of spectral rendering engine instead of an RGB-based renderer. Geisler-Moroder and Dürs validation of spectral rendering (versus an RGB render) was of real-world interior scenes illuminated by artificial light sources.

In the field of architecture and daylighting, Inanici et al. (2015) used Ruppertsberg and Bloj's N-step method for multi-spectral simulations, to determine circadian lighting. They analysed interior scenes for circadian and photopic lux using spectral skies from measured correlated colour temperature (CCT) values. To make the N-step method more available to architects and lighting designers, Inanici et al. (2015) released LARK - a grasshopper plugin (McNeel et al., 2010) for spectral simulations to evaluate circadian lighting (Inanici and ZGF Architects, 2015).

ALFA (Adaptive Lighting for Alertness) is also a $R a$ diance based multi-spectral simulation platform to compute circadian lighting. ALFA performs simulation on 81-colour channels, whereas LARK is set up to run a maximum of 9-channel simulations (Solemma, 2018).

LARK and ALFA are the two currently user-friendly programs to perform multi-spectral, physically accurate daylight simulations. They are both based on Radiance and is operated through the popular 3D modelling program for architects - Rhinoceros 3D (McNeel et al., 2010). Hence, in this research, the authors use LARK and ALFA to perform multi-spectral simulations of complex urban scenes under measured and computed spectral skies respectively. While previous studies have deduced colorimetric accuracy of simplistic scenes of objects and indoor scenes under artificial lighting, this study of urban environments in multi-spectral simulations under daylight will be first of its kind. 


\section{Methodology}

Working in the context of Singapore, the authors study three commonly occurring urban environments for their spectral characteristics.

- plaster: Environment enclosed with Housing Development Board (HDB) units, which feature plaster facades. $80 \%$ of the population in Singapore live in such units.

- green: Environment enclosed with trees and grass ground cover. $30 \%$ of the urban regions in Singapore are vegetation.

- reflective: Environment surrounded with reflective glass facade. Largely represents the commercial centres and office buildings of Singapore.

\section{Measuring the urban environments}

To represent each one of these urban spectral environments - plaster, green and reflective - the authors chose specific sites in Singapore that has two qualities. One that the materiality (plaster facades, vegetation and reflective facades) of the site encloses the space tightly with a narrow sky view factor; second, within 5 to 8 minutes of walk from the site, there is an open site with a large sky view factor to measure the sky spectra. The Figure 1 shows the sky view factor of the three chosen urban sites and its corresponding open site.
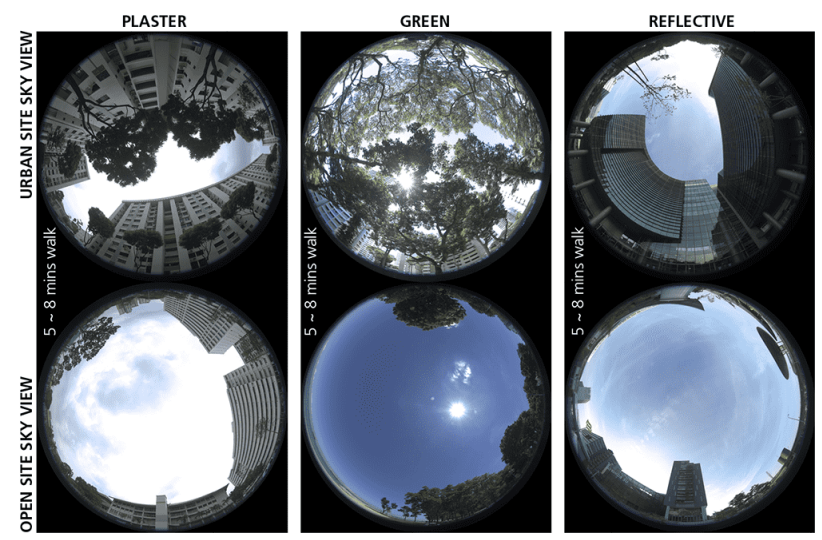

Figure 1: Urban site sky view and open site sky view for the three urban environments.

For each of the urban environments, High Dynamic Range (HDR) photographs as described by (Inanici, 2006) are captured using a Canon EOS 5D Mark III camera with a Canon equisolid projection fisheye lens (Canon EF 8-15mm f/4L USM). Vertical global spectral irradiance is measured using the Konica Minolta CL-500A illuminance spectrophotometer. The same equipment with a similar set up is used to measure the global sky spectral irradiance at the open site. Figure 2 demonstrates the horizontal and vertical measurement set-up at the open site and urban environment respectively. Measurements are taken under four sky conditions - morning (6:45 to 8:45 am), clear or intermediate, overcast and evening sky (18:00 to 19:30 pm). Morning and evening skies are categorised
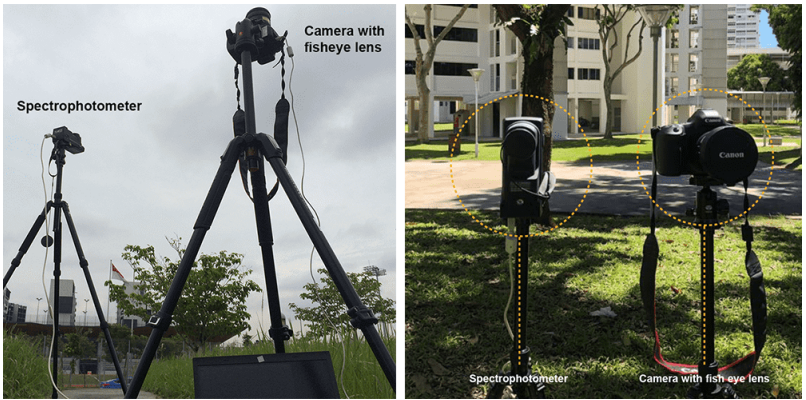

Figure 2: Left: horizontal measuring set up to record spectral measurements of sky at the open site. Right: vertical measuring setup to record spectral measurements within an urban environment.

based on the time rather than the sky conditions due to their relatively higher correlated colour temperature (CCT) than other sky conditions between 9:00 am to 18:00 pm. Note that morning and evening skies can also vary from clear, intermediate or overcast sky conditions. For each location, measurements are also taken in four directions to capture the effect of the different portions of the sky.

Each of the urban environment was also documented using a laser scanner (FARO FOCUS 3D X330). The cloud data from the laser scanner is used to build detailed 3D model of the environment in Rhinoceros 3D (McNeel et al., 2010). Material spectral reflectance data of the building finishes, ground cover, tree barks and leaves were measured using a Konica Minolta CM-2600d Spectrophotometer as described by Jakubiec (2016)

\section{Setting up spectral simulations}

LARK runs on the grasshopper plugin (McNeel et al., 2010) in Rhinoceros 3D (McNeel et al., 2010) and is an open-source programme. LARK is based on the $\mathrm{N}$-step algorithm and can currently run a 9-channel simulation. The inputs to LARK are the location, time, global horizontal solar irradiance, the measured spectral sky data for each scenario and the measured spectral surface reflectance for each of the surfaces in the 3D model. There are two Radiance sky generator program-gensky and gendaylit. LARK was initially set-up to use the gensky program to generate the sky spectral distribution. However, the authors edited LARK to run the gendaylit program for uniformity of comparison with the non-spectral daylight simulation that also uses the gendaylit program to generate the sky. Note, the sun in LARK is modelled as a nonspectral equal energy white source similar to the nonspectral simulations.

ALFA is a direct plugin to Rhinoceros 3D. The inputs to ALFA are - the 3D model, location, time, and measured material spectral reflectance. The spectral sun and sky in ALFA are precomputed in a radiative transfer library called libRadtran (Mayer and Kylling, 2005) for every location available in ALFAs database. The atmospheric profiles used 
Table 1: Summary of the spectral (LARK and ALFA) and non-spectral (PEREZ) simulation parameters.

\begin{tabular}{l|l|l|l}
\hline Parameters & LARK & ALFA & PEREZ \\
\hline Sky input & $\begin{array}{l}\text { Measured spectral sky irra- } \\
\text { diance, global solar irradi- } \\
\text { ance, location, time }\end{array}$ & $\begin{array}{l}\text { Pre-computed spectral sky } \\
\text { irradiance generated in li- } \\
\text { bRadtran, location, time }\end{array}$ & $\begin{array}{l}\text { Non-spectral and luminance } \\
\text { based sky, global solar irra- } \\
\text { diance, location, time }\end{array}$ \\
\hline Sun & $\begin{array}{l}\text { Non-spectral, equal energy } \\
\text { white source }\end{array}$ & $\begin{array}{l}\text { Non-spectral, equal energy } \\
\text { white source }\end{array}$ \\
\hline Atmosphere & N/A & $\begin{array}{l}\text { Sky spectra is computed us- } \\
\text { ing an AFGL atmospheric } \\
\text { profile }\end{array}$ & N/A \\
\hline Sky condition & $\begin{array}{l}\text { Determined by the global } \\
\text { horizontal irradiance input } \\
\text { to gendaylit program }\end{array}$ & $\begin{array}{l}\text { Users can choose from } \\
\text { overcast, hazy, heavy rain } \\
\text { clouds }\end{array}$ & $\begin{array}{l}\text { Determined by the global } \\
\text { horizontal irradiance input } \\
\text { to gendaylit program }\end{array}$ \\
\hline $\begin{array}{l}\text { Simulation for- } \\
\text { mat }\end{array}$ & $\begin{array}{l}\text { 91-channel rannel rectandaral RGB } \\
\text { Material Input }\end{array}$ & $\begin{array}{l}\text { Spectral material re- } \\
\text { flectance } \\
\text { flectance }\end{array}$ \\
\hline
\end{tabular}

in libRadtran to generate spectral sun and sky for ALFA is the U.S. Air Force Geophysics Laboratory's (AFGL) standard mid-latitude summer profile (Anderson et al., 1986). ALFA also allows the user to choose between a sky condition (clear, overcast, hazy, heavy rain clouds) and a ground spectrum.

For comparisons between spectral and widely used non-spectral simulations, all the three urban environments are simulated in a non-spectral standard Radiance environment. The inputs to the non-spectral simulations are - detailed 3D models, location, time, and global horizontal solar irradiance. The sky input for non-spectral simulations is the all-weather Perez sky model (Perez et al., 1993) based on luminance distributions. For all further comparisons presented in this paper, non-spectral simulations are referred to as PEREZ simulations hereafter. Materials assigned in PEREZ simulations are the measured spectral reflectance data converted to Radiance RGB material data.

The global solar horizontal irradiance used for LARK and PEREZ simulations are taken from the weather station at Singapore University of Technology and Design (SUTD), located in the east of Singapore. ALFA does not take the global irradiance as an input.

Due to certain limitations of representing materials in simulations, leaves of tree crowns in the green environment are assigned as an opaque (Radiance) plastic material type without any transmittance. Tree crowns are measured for their gap percentages and modelled as described by Balakrishnan and Jakubiec $(2016,2018)$. The reflective glass facade in the reflective environment is assigned as a Radiance metal material type. In reality, the facade is a reflective glass material that both transmits and reflects light.

Table 1 summarises the simulation parameters of LARK, ALFA and PEREZ.

\section{Results}

\section{Visual Comparisons}

Figure 3 demonstrates the visual difference between renders of spectral and non-spectral simulations. Colours in the non-spectral render (PEREZ) appear to have a yellow or brown tinge because of the lack of blue colour in its simulated sky.

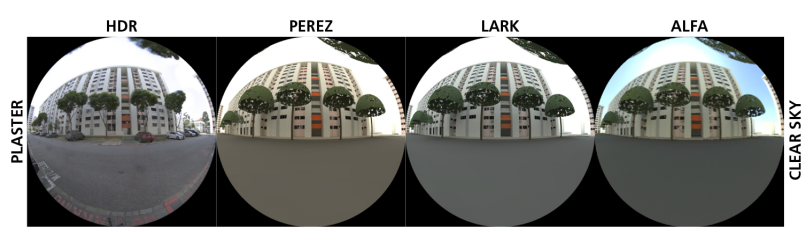

Figure 3: Comparison of plaster urban environment renders of spectral and non-spectral simulations with HDR photographs.

Direct Light: Direct light is the light from the sun illuminating the urban environments during the day. Figure 4 show the effect of direct light in the different urban scenarios and their respective renders using spectral (LARK and ALFA) and non-spectral (PEREZ) simulations under a clear sky. ALFA renders direct light warmer in colour than LARK. This effect is evident in both the plaster and green environment shown in Figure 4. The facade in the plaster environment is chosen as it directly sees the sun and is illuminated by direct light from the sun. In the green environment, the grass ground plane receives direct light from the sun which is reflected towards the tree crowns. Thus explaining why the grass ground plane and tree crowns in ALFA's renders appear warmer in tone than that of LARK's in the green scene (Figure 4). Effect of reflected light in a scene is pronounced only with the presence of a strong direct light source like the sun (Gurney, 2010). Hence the illumination effects under direct and reflected light on a clear day is significantly influenced by the simulated colour of the sun. In ALFA the sun is modelled us- 


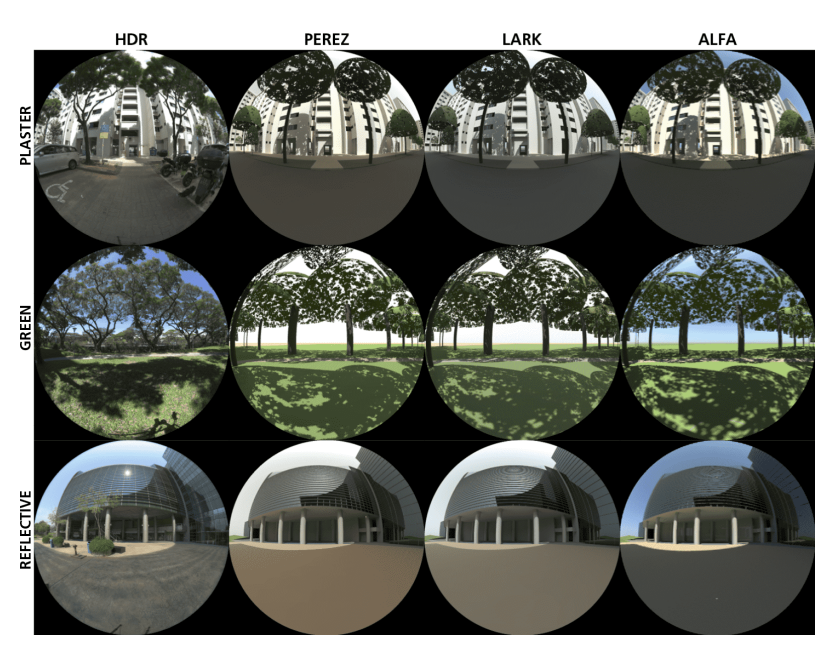

Figure 4: Comparisons of the effects of direct light in HDR photograph, non-spectral PEREZ render, spectral $L A R K$ and ALFA renders of different urban environments under clear sky conditions.

ing the extraterrestrial solar spectrum and travelling through an atmospheric profile. However, in LARK the sun is modelled as a non-spectral equal energy white source without an atmosphere. Therefore, direct light in ALFA is warmer, has richer colour information and varies based on its position in the sky.

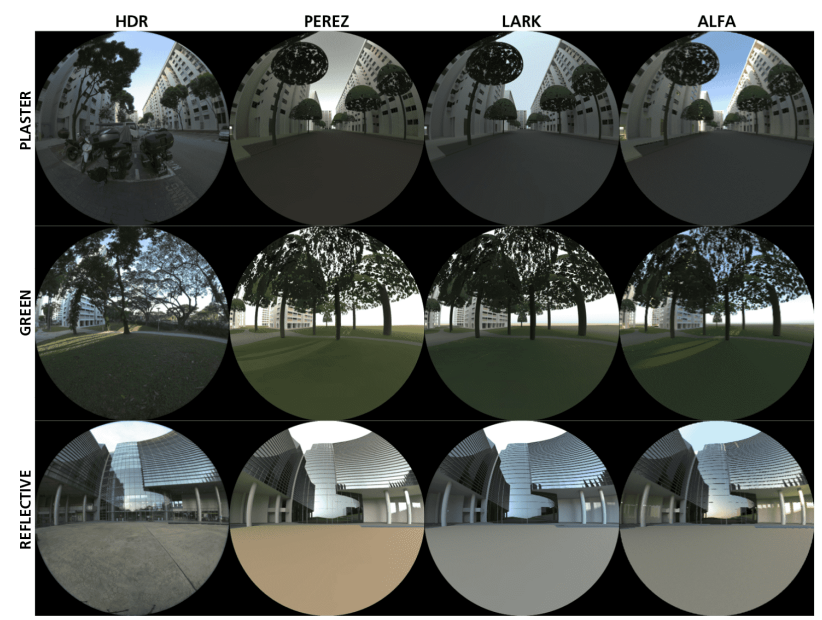

Figure 5: Comparison of the effects of direct light between HDR photograph, non-spectral PEREZ render, spectral $L A R K$ and $A L F A$ renders under evening sky conditions.

Shadows in a scene are also a result of a direct light source. Bluer the diffuse sky opposite the sun the darker and bluer are the shadows cast. With clouds in the sky, the shadows become greyer (Gurney, 2010). Given that ALFA's spectral skies are based on a mid-latitude summer atmospheric profile, it's simulated diffuse sky is bluer than that of LARK. Therefore ALFA renders the deepest shadows compared to PEREZ and LARK and is particularly evident in the dappled shadows of the green scene in Figure 4.

Reflective facades that face the sun on a clear sky throw specular reflections of the sun as seen in the HDR photograph of the reflective environment, in
Figure 4. However, this effect does not appear in any of the spectral or non-spectral renders.

The low-angle sun is the direct source of light in morning and evening clear skies. Colours of the lowangle direct light source vary based on atmospheric constituents and distance travelled through the atmosphere. In general, they are warmer with an orange tint compared to when the sun is higher in the sky. The HDR photographs in Figure 5 demonstrate the effect of low-angle direct light from an evening sky. An upper streak of yellow-orange light is seen in the HDR photograph of the plaster facade and on the ground of the green environment. ALFA is able to render these streaks with warm-orange in both the environments. In the PEREZ simulation, the streak is rendered as white light in the plaster scene and slightly yellowish in the green scene due to the interreflections of the surrounding. For the reflective environment, the reflection of the sun can be seen at the bottom of the facade as a visible yellow-orange reflection in ALFA and white in PEREZ renders. LARK is currently unable to render the effect of the low-angle sun either in the morning or evening.

Diffuse Skylight: When there is a clear or an intermediate sky, the part of the sky dome that does not contain the sun, provides the diffuse skylight. Contrary to direct light, the diffuse skylight is a soft non-directional light. Depending on the spectra and sky condition the colour of the diffuse skylight can change.

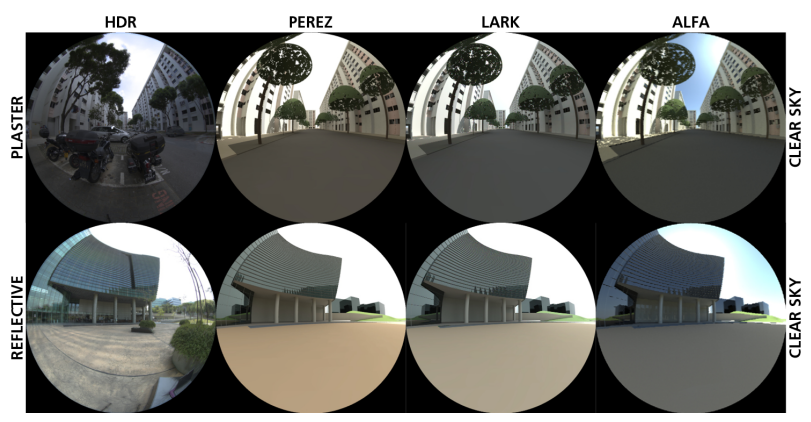

Figure 6: Comparison of the effects of diffuse skylight between HDR photograph, non-spectral PEREZ render, spectral LARK and ALFA renders under clear sky.

Figure 6 illustrates the effect of the diffuse skylight in the plaster and reflective environment on a clear day. The directions of the environment in Figure 6 are specifically chosen because they face the diffuse sky (bluest part of the sky) opposite the sun. The facade on the right in the plaster environment is illuminated by the diffuse skylight. Visually, the renders in ALFA seems to capture the effect of the diffuse sky in the plaster and reflective environment than LARK's renders.

However, under the morning sky, both LARK and ALFA equally renders the effect of the diffuse sky in 
both the environments shown in Figure 7. Though the reflection of the yellow-orange sun appears only in the ALFA renders. The spectral sky in LARK

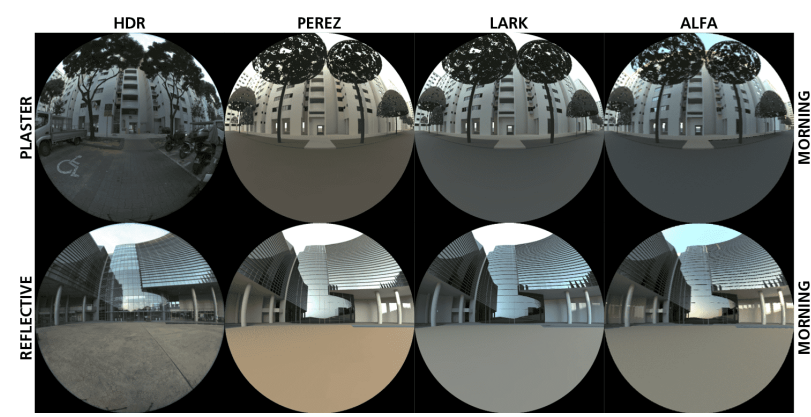

Figure 7: Comparison of the effects of diffuse skylight between HDR photograph, non-spectral PEREZ render, spectral LARK and ALFA renders under morning sky.

is modelled as an average distribution of the measured spectral sky data, including the sky and the sun. The non-spectral sun is then added to the skydome. As a result, LARK currently underpredicts the blueness of the skydome for clear skies. However, for the morning/evening sky the measured spectral irradiance represents the spectra of the skydome with minimal contribution from the low-angle sun. Moreover, in LARK, the low-angle sun is not modelled. Given these two reasons, renders in LARK illuminated by the diffuse morning and evening sky represent richer colour information. Renders in PEREZ under morning and evening skies (Figure 7) significantly misrepresents the colour information.

Diffuse Overcast: Overcast or heavily clouded skies provide a softer diffuse light for illumination without sharp shadows and contrasts. Figure 8 shows the comparison between spectral and non-spectral renders with the HDR photographs.

The global horizontal irradiance determines whether the sky is overcast or not. It is an input to generate the sky in LARK and PEREZ. The irradiance measurements can vary from the time the spectral measurement is taken at the urban scene to the open sky measurement. This variation leads to errors - faint speckled shadows of trees - in simulations as seen in the plaster environment of Figure 8. The HDR photograph of the plaster facade in Figure 8 was captured when the sky was heavily clouded. At the open site, the sun was obscured partially. Hence a higher global horizontal irradiance was recorded and used to run the simulations in LARK and PEREZ.

This error in ALFA is avoided as the user can specify the sky condition to perform the simulations. Hence, in all the heavily clouded sky conditions simulated in ALFA the sky is defined as overcast. However, ALFA's atmospheric profile for an overcast sky renders scenes with a slight yellow tint. Measurements of global sky spectra accurately represent a uniform sky such as an overcast sky. Hence, LARK's colour information in renders under overcast skies appear closer to the HDR captures.

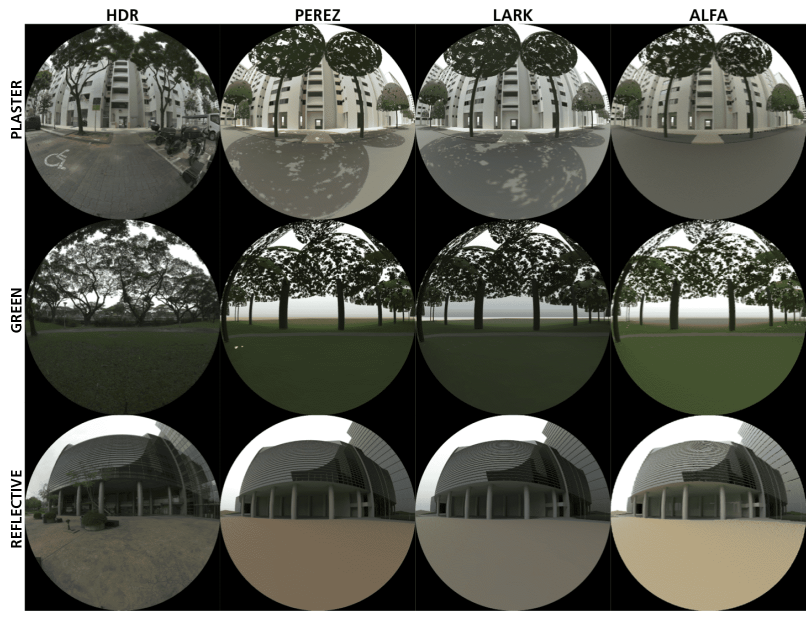

Figure 8: Comparison of the effects of diffuse overcast light between HDR photograph, non-spectral PEREZ render, spectral $L A R K$ and $A L F A$ renders.

\section{Spectral Comparisons}

In this section, the authors present a comparison of the measured spectral irradiance taken on site with the spectral outputs from LARK and ALFA. Spectral irradiance measurements were taken vertically with the illuminance spectrophotometer shown in Figure 2, in four directions and different sky conditions for the various locations. Measurements using the spectrophotometer are referred to as sensor hereafter in this paper.

The spectral irradiance measured using the sensor is at every $1 \mathrm{~nm}$ interval. ALFA's spectral irradiance outputs are at every $5 \mathrm{~nm}$ interval from $380 \mathrm{~nm}$ to 780 nm. However, LARK's spectral irradiance outputs are 9 values, one for each of the discrete 9 channelbins between $380 \mathrm{~nm}$ to $780 \mathrm{~nm}$ (Inanici et al., 2015). For comparison purposes, LARK's spectral irradiance measures are linearly interpolated at intervals of 5 $\mathrm{nm}$. The plots of the comparisons is available online as a Github repository (Balakrishnan, 2019).

To calculate the errors of the spectral distribution produced by ALFA and LARK with the sensor measurements, the Root Mean Square Error (RMSE) is computed as per the equation (1). As the main focus is to compare the spectral distribution rather than the absolute spectral irradiance values, the RMSE is computed for normalised spectral irradiance values. Normalisation for every spectral distribution is obtained by having a value of 1 at $560 \mathrm{~nm}$.

$$
\operatorname{RMSE}=\frac{1}{n} \cdot \sqrt{\sum_{j=1}^{n} \frac{I_{\text {sim }}^{j}-I_{\text {Sensor }}^{j}}{I_{\text {Sensor }}^{j}}}
$$

$I_{\text {sensor is the normalised spectral irra- }}^{j}$ diance measured using the sensor at the 
$j$-th tabulated wavelength, from the set $\{400,405, \ldots, 695,700\}$.

$I_{\text {sim }}^{j}$ is the normalised spectral irradiance resulting from either LARK or ALFA simulations at the $j$-th tabulated wavelength, from the set $\{400,405, \ldots, 695,700\}$.

Figure 9 illustrates the RMSE errors, calculated using the equation (1), for simulation outputs in LARK and ALFA under the four sky conditions (clear, morning, evening and overcast) and the three urban environments (plaster, green, reflective.)

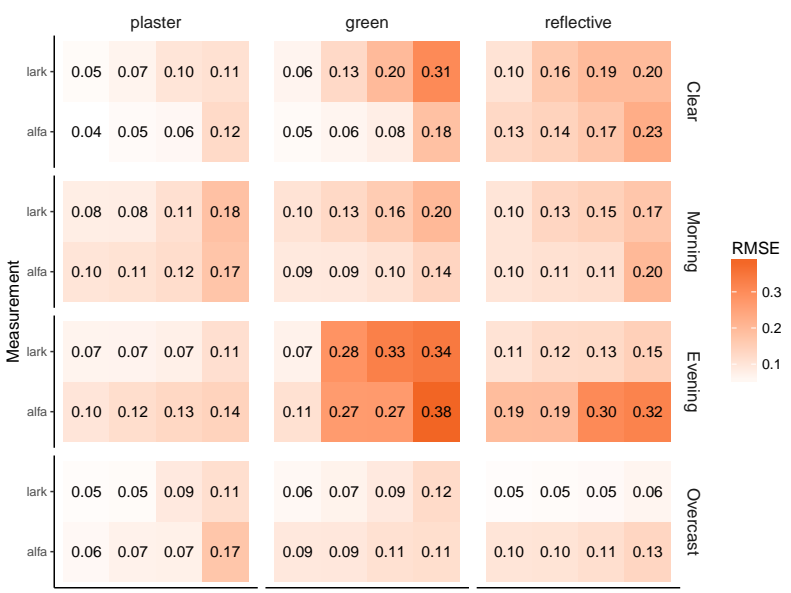

Figure 9: RMSE errors calculated as per equation (1) for LARK and ALFA multi-spectral simulations under the four sky conditions in plaster, green and reflective environments.

RMSE variation in different urban environments: Among the three environments, LARK and ALFA have lower RMSE ranging from 0.04 to 0.18 in the plaster environment. The relative accuracy of the 3D model and material assignment in the plaster environment is the main reason for this lower RMSE. In the plant environment, the RMSE ranges from 0.06 to 0.38 , with the most significant error caused due to the non-inclusion of low-lying shrubs in the 3D model. The RMSE range in the reflective environment is from 0.05 to 0.32 . Assigning the sky condition as hazy in ALFA does not represent the measured cloudy evening sky. Thus, it is the cause for the largest RMSE in the reflective environment.

RMSE variation under different sky conditions: Spectral simulations with an overcast sky produced the least RMSEs (from 0.05 to 0.17) compared to other sky conditions. The largest RMSE of 0.17 was due to assigning a sky with a partially obstructed sun as an overcast sky. The range of RMSE for spectral simulations under clear skies is 0.05 to 0.31 . The 0.31 RMSE caused due to LARK's non-spectral equal energy white sun not reflecting warm light off the ground in the green environment. Environments simulated under morning skies has an RMSE range 0.08 to 0.20. The low-angle sun and sky in ALFA being reflected off the facade in the reflective environment cause the 0.20 error. Spectral simulations under the evening skies have the most substantial RMSE ranging from 0.07 to 0.38 . It is the inaccuracy of the geometric model in the plant environment and the hazy sky condition chosen to simulate the evening sky for the reflective environment in ALFA that caused the large RMSE.

RMSE variation between LARK and ALFA: LARK has lower RMSE than ALFA under uniform sky conditions where the effect of the sun is minimal, such as with overcast, evening and morning skies. Under these sky conditions, the global measured spectral irradiance represents the whole skydome. Here, the measured sky spectra input to LARK generates a sky that has closer colorimetric properties to the real sky. When the sun is present and there is a variation of colour across different parts of the skydome, the measured global spectral irradiance is only an average representation of the skydome. Therefore, with this measurement provided as an input to LARK, it is once again averaged to produce the spectral sky model reducing the stark contrasts occurring in different parts of the skydome. Under clear skies, ALFA returns lower RMSEs than LARK. Neither LARK nor ALFA can currently simulate clouds, thus both LARK and ALFA cause significant errors in scenarios where the sun is partially obscured.

\section{Colour Difference Comparisons}

To compare the rendered coloured output of the spectral and non-spectral simulations, the authors propose a colour difference comparison in the CIE $L^{*} a^{*} b^{*}$ colour space. The colour difference is the distance between two colours coordinates within a colour space. Distances calculated between two colours in the three-dimensional RGB space do not relate to human perceived colour difference (Jakubiec, 2016). However, distances in $\mathrm{L}^{*} \mathrm{a}^{*} \mathrm{~b}$ colour space, are perceptually related and hence make it a better colour space to compute the colour difference between pixels of the renders - PEREZ, ALFA and LARK - from HDR photographs.

Brightness among the rendered images and with the HDR photographs can vary due to differences in the simulated versus actual sky brightness. Therefore, to compare the absolute differences in colour, the colour difference $(\Delta E)$ is computed as in equation (2) excluding the $L^{*}$ of the $L^{*} a^{*} b^{*}$ colour space.

$$
\Delta E=\sqrt{\left(a_{1}-a_{0}\right)^{2}+\left(b_{1}-b_{0}\right)^{2}}
$$

$a_{0}$ is the chromacity value in the green-red axis of the reference colour (pixels of the HDR photograph). $a_{1}$ is the chromacity value in the green-red axis of the target colour (pixels of LARK, ALFA or PEREZ simulations). $b_{0}$ is the chromacity value in the blueyellow axis of the reference colour (pixels of the HDR 
Table 2: Colour difference comparisons.

\begin{tabular}{l|l|l|l|l}
\hline Light & Location & $\begin{array}{l}\text { PEREZ } \\
(\Delta E)\end{array}$ & $\begin{array}{l}\text { LARK } \\
(\Delta E)\end{array}$ & $\begin{array}{l}\text { ALFA } \\
(\Delta E)\end{array}$ \\
\hline \multirow{2}{*}{ Direct } & plaster & 2.3 & 1.4 & 2.4 \\
Light & plant & 7.1 & 4.0 & 9.6 \\
& reflective & 1.6 & 3.6 & 0.8 \\
\hline \multirow{3}{*}{ Shade } & plaster & 2.0 & 1.6 & 1.7 \\
& plant & 4.4 & 6.5 & 4.5 \\
& reflective & 13.1 & 9.0 & 16.1 \\
\hline \multirow{2}{*}{ Diffuse } & plaster & 3.9 & 1.3 & 4.0 \\
overcast & plant & 3.9 & 2.6 & 4.4 \\
& reflective & 2.0 & 1.8 & 2.1 \\
\hline \multirow{2}{*}{ Diffuse } & plaster & 8.8 & 5.3 & 5.3 \\
skylight & plant & 4.5 & 1.5 & 3.1 \\
& reflective & 4.7 & 1.9 & 0.6 \\
\hline
\end{tabular}

photograph). $b_{1}$ is the chromacity value in the blueyellow axis of the target colour (pixels of LARK, ALFA or PEREZ simulations).

All comparisons are performed using the default colour setting of the simulation outputs (Radiance RGB) and HDR image capture (sRGB). While the exposures are adjusted to be uniform among the renders and with the HDR photographs, no further calibrations for colour (such as tone mapping) have been applied.

To categorically compute the colour differences, scenarios are selected, one direction per location (plaster, green and reflective) that best represents the effect of direct light (light from the sun), shade (when direct light falls on surfaces and causes shadows), diffuse overcast light (light from an overcast sky) and diffuse skylight (light from the diffuse sky). A region of 5 $\mathrm{x} 5$ pixels illuminated by the categorised light effect (direct, shade, diffuse overcast, diffuse skylight) is selected among all the renders and HDR photographs for comparisons. The Figure 10 is an example illustration of pixel selection for comparisons under direct light in the plaster environment/location.

Table 2 gives a summary of colour differences $(\Delta E)$ among the renders of the three simulations under the different lights. Spectral renders of LARK have the smallest $\Delta E$ for most of the scenarios and in particular under diffuse overcast light. ALFA's spectrally rendered pixels have the lower $\Delta E$ values for reflective facades under all the different light scenarios except in shade where the difference is quite high. The simulated sky in ALFA is 'bluer' than LARK's, and this difference is more evident under direct light from clear skies. This is the reason why ALFA's renders are a closer match to the HDR captures in the reflective environment under clear skies.

As expected non-spectral renders of PEREZ have the largest $\Delta E$ for all the scenarios except for the grass pixel in the shade (Figure 4). The grass material is not accurately represented in simulations. Onsite the grass surface is a mix of green grass and earth. However, when measuring material properties of the grass surface, the authors took measurements of solely the grass. Hence a slightly yellow-green grass under shade, rendered in PEREZ, appears closer to the colour in the HDR photograph.

The difference in $\Delta E$ among the different rendering platforms is directly linked to how the colour of the sky and sun in each of the platform is rendered. In most of the cases, LARK's renders have the closest colour values (channel $\mathrm{a}^{*}$ and $\mathrm{b}^{*}$ ) to the HDR photograph as its sky input is measured global spectral irradiance. In sky conditions where the effect of the sun and the atmospheric transfer of light plays a vital role in the colour of the sky (such as clear skies) and the material reflects the sky (such as reflective facade) ALFA's render's match closely in colour to the HDR photograph.

\section{Conclusions}

LARK and ALFA are the two most accessible and easy to use interfaces for the daylight community to perform spectral daylight simulations. They both were created to compute circadian lighting. However as shown in this paper, both platforms can produce spectral renders that have richer colour information of the context and represent physically accurate colour perceptions when compared to non-spectral standard RGB daylight simulations.

A summary of the different parameters used in ALFA and LARK is listed in Table 1. LARK and ALFA mainly differ in the way the sun and sky are represented. While LARK can take measured global spectral sky irradiance as an input, it lacks an atmospheric profile found in ALFA. Without an atmospheric profile, colour renditions of the low-angle sun in the sky cannot be depicted. ALFA's atmospheric profile is currently based on a generic mid-latitude summer sky. ALFA has the potential to improve the accuracy of colour renditions and spectral outputs by including location-specific atmospheric profiles.

Apart from measured spectral sky input LARK can also take Correlated Colour Temperature (CCT) of the sky as an input. If CCT is used, it has to be converted to spectral irradiance data based on the definitions of CIE standard illuminants (Wyszecki and Stiles, 1982; Inanici et al., 2015). Measured spectral irradiance data gives more accuracy than the standard sky conditions represented by the CIE illuminants. Hence the authors have published a public Github repository (Balakrishnan, 2018) where measured global spectral irradiance data for different equatorial skies can be downloaded.

In LARK simulations, the modelled spectral sky is an average distribution of the measured spectral sky data including both the sun and the sky when the sun is present. An equal energy white sun is added, reducing the spectral contribution of the diffuse skylight in 


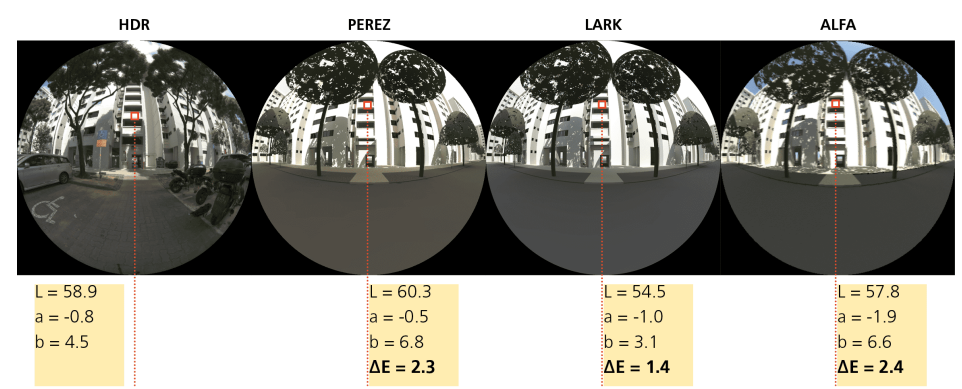

Figure 10: Example of colour difference comparison of pixels in direct light at the plaster location.

a clear sky render. An update to LARK weighing the CCT of the sun and sky based on the direct and diffuse global horizontal irradiance is in progress by the authors with the original developers.

This paper presents a first-of-its-kind framework to compare spectral daylight platforms for their visual, spectral and colorimetric accuracy of complex daylight scenes. These platforms are essential to represent complex architectural and urban scenes using appearance-sensitive daylight renders respecting local climate and context. In this work, the specifications of LARK and ALFA which affect the visual appearance of spectral renders are compared to provide users and developers with a deeper understanding and greater applicability of multi-spectral daylight simulations in general.

\section{References}

Anderson, G. P., S. A. Clough, F. Kneizys, J. H. Chetwynd, and E. P. Shettle (1986). Afgl atmospheric constituent profiles $(0.120 \mathrm{~km})$.

Balakrishnan, P. (2018). Equatorial skies. https://github.com/prijibalakrishnan/ equatorialskies.

Balakrishnan, P. (2019). Spectral comparison plots. https://github.com/prijibalakrishnan/ spectralirradiance.

Balakrishnan, P. and J. A. Jakubiec (2016, sep). Measuring light through trees for daylight simulations: A photographic and photometric method. In Proceedings of the 3rd IBPSA-England Conference BSO 2016, pp. 115 - 122. IBPSA.

Balakrishnan, P. and J. A. Jakubiec (2018). Program to generate simple tree models for lighting simulations. https://github.com/C38C/gen_tree.

Devlin, K., A. Chalmers, A. Wilkie, and W. Purgathofer (2002). Tone reproduction and physically based spectral rendering. Eurographics.

Geisler-Moroder, D. and A. Dür (2009). Colorrendering indices in global illumination methods. Journal of Electronic Imaging 18(4), 043015.

Gurney, J. (2010). Color and light: A guide for the realist painter, Volume 2. Andrews McMeel Publishing.
Inanici, M. (2006). Evaluation of high dynamic range photography as a luminance data acquisition system. Lighting Research \& Technology 38(2), 123134 .

Inanici, M., M. Brennan, and E. Clark (2015). Spectral daylighting simulations: Computing circadian light. In Proceedings of BS2015: 14th Conference of International Building Performance Simulation Association, Hyderabad, India, pp. 1245-1252.

Inanici, M. and L. ZGF Architects (2015). Lark spectral lighting.

Jakubiec, J. A. (2016). Building a database of opaque materials for lighting simulation. In PLEA 2016Proceedings of the 32nd International Conference on Passive and Low Energy Architecture.

Mayer, B. and A. Kylling (2005). The libradtran software package for radiative transfer calculationsdescription and examples of use. Atmospheric Chemistry and Physics 5(7), 1855-1877.

McNeel, R. et al. (2010). Rhinoceros version 5.0. Service Release SR14 22 July $201 \%$.

McNeel, R. et al. (2010). Grasshopper generative modeling for rhino. Computer software (2011b), http://www.grasshopper3d.com.

Perez, R., R. Seals, and J. Michalsky (1993). Allweather model for sky luminance distributionpreliminary configuration and validation. Solar energy $50(3), 235-245$.

Ruppertsberg, A. I. and M. Bloj (2006). Rendering complex scenes for psychophysics using radiance: How accurate can you get? JOSA A 23(4), 759768 .

Solemma (2018). Alfa - adaptive lighting for alertness.

Ward, G. J. (1994). The radiance lighting simulation and rendering system. In Proceedings of the 21st annual conference on Computer graphics and interactive techniques, pp. 459-472. ACM.

Wyszecki, G. and W. S. Stiles (1982). Color science, Volume 8. Wiley New York. 